Post-mortem Imaging Adjudicated Sudden Death: Causes and Controversies

Giuseppe Femia ${ }^{1,3}$, Christopher Semsarian ${ }^{1,3,4}$, Neil Langlois ${ }^{5}$,

Mark McGuire ${ }^{1,3}$, James Raleigh², Andrew Taylor ${ }^{6,7}$,

Rajesh Puranik ${ }^{1,3}$

1. Department of Cardiology, Royal Prince Alfred Hospital, Sydney, Australia

2. Department of Radiology, Royal Prince Alfred Hospital, Sydney, Australia

3. Faculty of Medicine, University of Sydney, Sydney, Australia

4. Agnes Ginges Centre for Molecular Cardiology, Centenary Institute, Sydney, Australia

5. School of Anatomy and Physiology, University of Adelaide, Frome Road, Adelaide, South Australia

6. UCL Institute of Cardiovascular Science, London, UK

7. Great Ormond Street Hospital for Children, NHS Foundation Trust, London, UK 


\subsection{Introduction}

Sudden cardiac death $(S C D)$ is an unexpected and devastating tragedy associated with major implications for the surviving family and community (1). SCD can be defined as a sudden pulse- less condition in the absence of a non-cardiac condition (2). The estimated annual incidence for those aged 1-35 years is 0.5 to 3.2 per 100000 person years with those aged 31 to 35 years having the highest incidence (3.2 per 100000 person years) (3) (4). The life time risk of SCD in the adult population greater than 45 years of age ranges from $2.4-3.4 \%$ for women and $6.7-11.2 \%$ for men (5). For the adult population, the most frequent underlying cause of SCD is coronary artery disease (CAD) and cardiomyopathy. For the younger population, the causes of SCD are diverse and may be categorised into structural (e.g. inherited cardiomyopathies and Marfan syndrome) and non-structural, regarded as arrhythmogenic conditions (Brugada, long and short QT syndromes and catecholaminergic polymorphic ventricular tachycardia) (6) (7).

The post-mortem or conventional autopsy is widely considered the "gold standard" in delineating the cause of SCD. In the majority of SCD cases attributable to a cardiac cause, structural heart disease is detected by conventional autopsy (8). In contrast, the cause remains unclear in approximately $40 \%$ of young SCD cases, despite detailed histopathological and toxicological analyses (3). Despite its proven capabilities in post mortem diagnostics, there is a growing reluctance amongst families and some health care professionals to refer for conventional autopsies and as a result, the rate of autopsy referrals has declined globally (9). For reportable deaths, coroners must reconcile the public interest and potential value to the family of the deceased being fully investigated balanced against consideration of specific cultural and religious customs. Hence, there is an emerging role for an alternative, non-invasive post-mortem examination.

Recently, the emergence of post-mortem computed tomography (pmCT) and post-mortem magnetic resonance imaging (pmMR) addresses the need for a non-invasive and expeditious post-mortem investigation that maintains diagnostic accuracy. For both pmCT and pmMR, there have been substantial improvements in imaging technology, image acquisition and processing domains. When compared to conventional autopsy, a number of studies have shown promising evidence in support of post-mortem imaging. In some situations, the 
information obtained from post-mortem imaging or autopsy could be complimented by genetic testing. In both autopsy positive and negative cases, the addition of genetic testing may add information to identify the cause of death or provide an opportunity to screen family members for inheritable conditions (3) (10). In this review, we will discuss the potential and emerging roles for post-mortem imaging in the diagnosis of SCD in children and adults.

\subsection{Causes of Sudden Cardiac Death}

The causes of SCD can be divided into two categories: inherited and acquired. Inherited conditions may be further characterised as structural or arrhythmogenic. Structural conditions may be genotype and/or phenotype positive and include the inherited cardiomyopathies, such as hypertrophic cardiomyopathy (HCM), dilated cardiomyopathy (DCM), arrhythmogenic right ventricular cardiomyopathy (ARVC) and Marfan syndrome. The inherited arrhythmogenic syndromes are characteristically genotype positive but phenotypically have structurally normal hearts. These conditions include Brugada syndrome (BrS), long QT (LQTS) and short QT syndromes (SQTS) and catecholaminergic polymorphic ventricular tachycardia (CPVT). Acquired causes of SCD include myocarditis and CAD. Noninherited structural conditions also include coronary artery anomalies as a cause for SCD The most common cause of SCD in those less than 35 years of age is 'un-ascertained, presumed primary arrhythmogenic disorder" where the heart is structurally normal (7). For those older then 35 , the most common cause of SCD is ischemic heart disease (11). That said, structural conditions account for about $50 \%$ of deaths in those less than 5 years of age and $75 \%$ for those aged between 5 and 35 years of age.

\subsection{Conventional Autopsy}

Conventional autopsy involves an extensive and thorough external examination as well as an in-depth assessment of internal organs with blood and tissue sampling for histological, microbiological and toxicological examination. The Coronial process, including detailed autopsy can be long and arduous, potentially adding substantial emotional stress to families as well as postponing funeral and religious ceremonies. Religious and cultural groups have different views on autopsy with some being opposed to the practice. As such, the referral rate for conventional autopsy has declined, particularly for the fetal and pediatric 
populations (12). In addition to the reduction in referrals, several issues deplete the accuracy of conventional autopsy including the variability in tissue sampling practices and the lack of standardized examination protocols across forensic facilities.

Given the current sentiment regarding conventional autopsies, there is a growing need to develop alternative strategies for the investigation of sudden and unexpected death. One strategy uses post-mortem imaging as a 'triage' tool to determine the need for further, more conventional, histo-patholological examination. In some cases, imaging may provide a cause of death without the need for an invasive autopsy. One group performed whole-body CT scans and external body examinations on 318 consecutive cases and found CT scanning has advantages in cases of accidental or suicidal death (13). In these cases, a lower proportion of cases proceeded to formal autopsy (31\%-50\%) than usual. Another strategy being tested is a minimally invasive autopsy facilitated by post-mortem imaging. In a recent study, full body pmMR at 1.5-Tesla (T) in conjunction with a minimally invasive autopsy (blood sample without dissection or tissue histology) was compared to a conventional autopsy in fetuses and children aged less then 16 years of age (14). In total there were 400 cases, where congenital $(\mathrm{N}=89 ; 22 \%)$ and infective/inflammatory $(\mathrm{N}=82 ; 21 \%)$ pathologies accounted for the most common diagnoses. A minimally invasive approach had excellent concordance for detection of major pathological abnormalities (89.3\%) related to death compared to conventional autopsy but there was some variation amongst age groups. In addition, with a minimally invasive approach, a full autopsy may not have been necessary in 165 cases (41\%); in these cases, the concordance rate between the two approaches was 99.4\%. Minimally invasive autopsy had the highest concordance rate in fetuses and newborns; $94.6 \%$ for fetuses at 24 weeks' gestation, $81 \%$ for fetuses more than 24 weeks' gestation, $81 \%$ for newborns aged 1 month or younger and $84.9 \%$ for infants from 1 month to 1 year. However, the minimally invasive approach was less accurate (53.6\%) for children from 1 year to 16 years of age. Using the pmMR and minimally invasive autopsy approach, sepsis was the most commonly missed cause of death (24 of 83 cases; $29 \%$ ). In terms of cardiac pathologies (with or without relation to death), the combined approach failed to detect 4 cardiac conditions in the fetal group (Tetralogy of Fallot (ToF), ventricular septal defect (VSD) and myocardial infarction) and 6 in the older group (myocarditis). In addition, 10 cardiac abnormalities were incorrectly diagnosed in the fetal group (aortic stenosis, 
anomalous venous drainage, co-arctation of aorta and VSD) and 2 in the children group (ASD). Using pmMR alone, the concordance rate with conventional autopsy was lower than the combined approach (55.5\%) with sepsis and placental pathology most commonly missed by $p m M R$. This study suggests that pmMR combined with a minimally invasive autopsy may provide a reliable alternative to conventional autopsy, especially in those cases aged under 1 year.

\subsection{Post-mortem Imaging - Current Evidence for Determining Cause of Death}

Post-mortem imaging is a non-invasive tool that complements the conventional armamentarium and aids in the diagnosis of SCD. Both pmCT and pmMR are capable of high resolution cross sectional imaging with established utility in the post-mortem setting. Modern CT scanners have up to 320 slices and provide excellent spatial resolution however many post-mortem facilities only have access to 16 or 32 slice scanners limiting the technical quality and image resolution. The lower image resolution particularly becomes an issue when attempting to visualize small caliber vascular anatomy such as the coronary arteries (15) . In comparison to $\mathrm{pmCT}$, pmMR does not use radiation but instead relies on strong magnetic fields and radiofrequency energy to alter the spin of protons within tissue. The image quality of MR depends on the signal to noise ratio and differs with various field strengths where current facilities use either 3-T or 1.5-T. Imaging at 3-T generates greater signal than at 1.5-T and can potentially provide higher resolution images with shorter acquisition times, making it ideal for visualising small bones, breast tissue, brain, spine and cardiac structures (16). Imaging at 3-T is however more susceptible to image artifacts and potentially more expensive than 1.5-T scanners (17). In terms of acquisition speed, CT is faster than MR and allows for whole body imaging within a few minutes. MR is also potentially dangerous in the presence of ferromagnetic foreign bodies such as "unsecured" cardiac devices or medical pumps. In these cases, caution is recommended, especially where such a device maybe free rather than fixed and thus be attracted directly to the magnet. Overall, pmCT has excellent visualization of the skeletal system, gas, fluids and foreign bodies while pmMR has excellent soft tissue contrast for most solid organs (16). These technical differences provide each modality with distinct strengths and weaknesses in post-mortem imaging, summarised in Table 1. 


\subsection{Post-mortem Imaging in Fetuses and Children}

There is an increasing reluctance amongst health care professionals and parents to refer fetuses and children for conventional autopsy. To address this issue, the development of alternative imaging strategies has become a priority, especially with sudden and unexpected deaths. As a diagnostic tool in this population, pmMR has been shown to be highly specific with a high negative predictive value. One study compared 3 dimensional pmMR images at 1.5-T to conventional autopsy from 185 fetuses younger than 24 weeks gestation, 92 fetuses older than 24 weeks gestation and 123 newborns and children younger than 16 years of age with SCD (18). Compared to autopsy, pmMR correctly diagnosed 19 of 22 (86\%) structural cardiac abnormalities from the pre-natal group and 13 of 13 (100\%) from the post-natal group but missed three cases of structural heart disease ( 2 ToFs and 1 VSD) in the fetal group and 8 cases of myocarditis in the post-natal group. In addition, pmMR overcalled 13 abnormalities. Overall pmMR had a sensitivity, specificity, positive predictive value (PPV) and negative predictive value (NPV) of $72.7 \%, 96.2 \%, 72.7 \%$ and $96.2 \%$, respectively for any cardiac pathology; the accuracy was even higher for major structural abnormalities. This study highlights the difficulty differentiating myocarditis from postmortem oedema using pmMR. Differentiating the two conditions poses a diagnostic dilemma and may rely on image guided biopsy to make a histological diagnosis.

In a prospective study comparing the diagnostic performance of perinatal and paediatric post-mortem imaging, 53 fetuses and 29 children underwent whole body pmCT using a 64slice multi-detector system and pmMR at 1.5-T prior to autopsy (19). Comparing pmCT and pmMR to conventional autopsy, pmCT was found to have limited value in young children and fetuses. In this group of patients, pmMR was superior to pmCT with a higher diagnostic accuracy (43.6\% vs. $32.7 \%)$. In addition, significantly more pmCT than pmMR examinations were non-diagnostic (18/82 (21.9\%) vs. $4 / 82(4.9 \%))$. This difference was enhanced in the fetal group less than 24 weeks gestation (40\% vs. 11\%). Overall in this cohort, pmMR was more accurate at detecting cardiac and central nervous system abnormalities.

A third study compared pmCT with a 64-slice scanner and pmMR at 1.5-T to conventional autopsy in 17 patients aged 1-35 years of age with sudden death (20). In this cohort, pmMR correctly identified all 12 (100\%) cases with positive findings at autopsy. In addition, 
pmMR had no false negative cases compared to three (25\%) cases with pmCT. However, pmMR incorrectly diagnosed one HCM case, one ARVC case and one pneumonia case; the misdiagnosis of HCM is a common issue described by others. In this population, pmMR was more accurate than pmCT and was found to be highly sensitive (100\%) with a high negative (100\%) and positive (80\%) predictive value.

Lastly, in a systematic review examining the diagnostic accuracy of pmMR, data was extracted from 9 studies that included 146 fetuses, 11 children and 24 adults (21). In accurately identifying the final cause of death, pmMR had a sensitivity and specificity of $69 \%$ and $95 \%$ in fetuses and $28 \%$ and $64 \%$ in children and adults. These studies suggest that pmMR is highly accurate for imaging structural heart disease in younger patients, but may be less suitable for investigating SCD in adults. As demonstrated by two studies in this review, pmMR may be less suited to diagnosing coronary artery disease (22) (23). Figure 1 demonstrates three conditions suitable for diagnosis with pmMR and confirmed on histopathology; acute myocardial infarction, ARVC and aortic dissection.

\subsection{Post-mortem Imaging in Adults}

Compared to the younger population, there is less published data for post-mortem imaging in the adult SCD population. The largest study to date $(N=182)$, compared whole body pmCT using a 16-slice scanner and pmMR at 1.5-T to conventional autopsy (24). Within this population, pmCT was the more accurate imaging modality with a higher concordance to conventional autopsy ( $68 \%$ vs $57 \% ; p=0.54$ ). The most common conditions resulting in diagnostic discrepancies between autopsy and imaging were CAD, pulmonary emboli, pneumonia and intra-abdominal lesions. Using autopsy, 86 deaths were attributed to CAD and 10 to pulmonary emboli however post-mortem imaging failed to diagnose 12 cases (14\%) of CAD and 10 cases (100\%) of pulmonary emboli. It is not surprising that these two conditions were the most frequently missed given the difficulty in diagnosing arterial occlusions and infarctions using the current post-mortem techniques. In their systematic review, Thayyil et al found that pmMR was less accurate in identifying the final cause of death for children and adults $(\mathrm{N}=35)$ compared to fetuses $(\mathrm{N}=146)$ with lower sensitivity and specificity; $28 \%$ and $64 \%$ vs. $69 \%$ and $95 \%$, respectively (14). The most common missed diagnosis in the adult population were coronary artery disease and acute myocardial 
infarction (22). Although the published data is limited to small, heterogeneous studies, they highlight the potential need for using different imaging modalities in different age groups (Table 1).

\subsection{Role of Post-mortem Imaging in the Investigation of Inherited Cardiac Causes of}

\section{Sudden Death}

The effectiveness of post-mortem imaging in the diagnosis of inherited cardiac diseases is variable depending on the underlying conditions. In these circumstances, there may be significant value from combining post-mortem imaging, genetic testing and more invasive investigations to assist in diagnosing the cause of death. We propose the following schema categorizing conditions based on the ability to make a diagnosis with post-mortem imaging; (i) definite phenotype, (ii) borderline phenotype or (iii) absent phenotype.

(i) Definite Phenotype: For certain inherited structural conditions such as syndromic aortopathies, ARVC and premature CAD with characteristic signs of familial hypercholesterolemia a definitive phenotype can often be identified with post-mortem imaging. In these cases, post-mortem imaging may prevent the need for more invasive investigations such as conventional autopsy. Securing an accurate clinical diagnosis in these cases provides an important opportunity to offer clinical screening and/or cardiac imaging to family members.

(ii) Borderline Phenotype: For some inherited conditions such as HCM, post-mortem imaging may have limited capabilities to confirm a diagnosis. The difficulty with diagnosing HCM with post-mortem imaging is differentiating pathological asymmetric myocardial hypertrophy from concentric left ventricular hypertrophy, physiological remodeling in athletes and post-mortem myocardial oedema leading to increased wall thickness. Establishing a diagnosis in this setting is critical as HCM is a genetically based condition with an autosomal dominant pattern of inheritance and so has implications for other family members who may be at risk. Further, HCM is the most common inheritable heart disease with a prevalence of 1 in 200 people and an important cause of SCD in the young (25) (26). Clearly in the scenario where there is clinical or imaging suspicion of HCM as the cause of 
SCD i.e. unexplained hypertrophy on post-mortem imaging, more invasive autopsy investigations and/or genetic testing maybe necessary to secure a diagnosis.

(iii) Absent Phenotype: Arrhythmogenic syndromes such as the channelopathies are most often associated with structurally normal hearts. This presents an important opportunity for post-mortem cardiac imaging to exclude structural heart disease i.e. relying on a good negative predictive value. In the situation where the heart can confidently be deemed structurally normal on post-mortem imaging, genetic testing maybe required to identify the cause of SCD. One common approach to genetic screening in this situation involves testing for four genes; KCNQ1 (LQTS1), KCNH2 (LQTS2), SCN5A (LQTS3 and BrS) and RYR2 (CPVT1) (29) (30). This testing regimen has shown variable success with a detection rate in selected tertiary referral populations up to $35 \%$ (31) (32). More recent advances in sequencing technologies have allowed the expansion of up to 200 detectable genes. Using genetic testing of 59 cardiac genes in the first ever prospective, population-based study of SCD in the young in Australia and New Zealand, a clinically relevant cardiac gene mutation was identified in $27 \%$ of autopsy negative cases (3). In SCD cases where genetic testing is necessary, it is recommended that such testing be undertaken in a specialized multidisciplinary clinic setting (33).

\subsection{Challenges for Post-mortem Imaging}

Despite encouraging results from the previously mentioned studies, post-mortem imaging has several limitations relating to image acquisition and access. In clinical practice, current protocols do not routinely use radio-opaque contrast for the visualization of vascular anatomy, mostly owing to the need for a spontaneous circulation. Without a contrast agent, the effectiveness of both pmCT and pmMR as diagnostic modalities are somewhat compromised. Calcification of coronary arteries can be detected by PmCT but without a radio-opaque contrast the modality is limited in its capacity to diagnose pathological coronary lesions namely soft plaque and/or luminal thrombus. In ante-mortem cardiac MR imaging, scar is detected with the administration of the contrast agent gadolinium however, without the diagnostic capabilities of gadolinium in pmMR certain cardiac conditions such as myocarditis and myocardial infiltration are difficult to accurately diagnose. 
Modern techniques are being established for post-mortem CT angiography (pmCTA) using various contrast agents and perfusion devices. One study selected 23 cases based on clinical history as well as the results of a baseline pmCT and evaluated the diagnostic value of pmCTA for investigating ischemic heart disease as the cause of SCD (34). The authors describe better visualisation of the coronary arteries and were able to better evaluate coronary lesions. Post-mortem CTA identified 11 of 14 coronary thrombi detected at autopsy with one identified as a chronic occlusion with collaterals and one as post-mortem clot. Despite studies demonstrating the utility of pmCTA in the diagnosis of ischemic heart disease, it remains a research tool and will require further investigation in order to become part of routine practice. One group has proposed a novel strategy that uses air as a negative contrast agent in addition to a conventional positive contrast agent for targeted pmCTA of the coronary arteries (35). In a small study $(\mathrm{N}=25)$ using a multi-detector $\mathrm{CT}$, this group described a simple and cost-effective targeted post-mortem cardiac angiography method.

Putrefaction and post-mortem cooling can alter the composition and structure of organs as well as the quality of post-mortem imaging. One particular issue is the variability that exists in the post-mortem interval to imaging as the precise time of death is often unknown. Changes in body temperature particularly due to cooling can substantially affect pmMR imaging by prolonging the $\mathrm{T} 1$ value and shortening the $\mathrm{T} 2$ value producing reduced signal to noise on both $\mathrm{T} 1$ and $\mathrm{T} 2$ sequences (36); $\mathrm{PmCT}$ is less susceptible to temperature changes above 0 degrees Celsius. These factors all contribute to making post-mortem imaging different from ante-mortem imaging and as a result, they require specialized image optimization and sequence modification. In addition, any diagnostic criteria of cardiac pathologies that relies on the difference between systolic and diastolic dimensions or functional abnormalities cannot be used. This does however present an important opportunity for the development of new diagnostic criteria in the post-mortem setting.

Although post-mortem imaging has been shown to be highly accurate in certain circumstances, access to scanners and reporting expertise remains challenging, particularly if the scanning facility is not co-located with a mortuary. In order to achieve specialized reporting, collaboration of local imaging facilities with trained professionals is 
recommended (37). Moving forward, standardized training programs may become necessary. Currently, there are no established protocols for post-mortem imaging but some image parameter recommendations are available but will often be modality and vendor specific (38). Of note the literature to date has often used different imaging modalities, acquisition protocols and reporting expertise that ultimately confounds conclusions from the available published data. The need for larger studies with uniform imaging protocols and reporting is necessary to further understand the diagnostic potential of post-mortem imaging in SCD.

\subsection{Conclusions and Future Directions.}

In the evaluation of $\mathrm{SCD}$, post-mortem imaging with either MR or CT may be combined with conventional autopsy and histological examination to identify the cause of death. Based on current knowledge, post-mortem imaging provides an effective alternative to conventional autopsy in specific circumstances. Post-mortem MR has important utility within the younger population especially with structural cardiac abnormalities. In contrast, post-mortem CT has an emerging role in the adult population, potentially assisting with the diagnosis of coronary artery disease. The addition of genetic testing in certain circumstances of suspected inherited heart disease may also add to overall diagnostic and ultimately screening capabilities.

In order for post-mortem imaging to become a routine component for the investigation of SCD, there remain important challenges of cost, resource availability and the development of specialized reporting and imaging protocols. However, in situations where conventional autopsy cannot be performed due to cultural or religious reasons or significant body decomposition, post-mortem imaging has definite advantages. More research is necessary to determine appropriate clinical pathways utilizing post-mortem imaging in the investigation of SCD. 


\section{References}

1. Key role of the molecular autopsy in sudden unexpected death. C, Semsarian and RM, Hamilton. 1, Jan 2012, Heary Rhythm, Vol. 9, pp. 145-150.

2. The incidence of primary cardiac arrest during physical activity. Siscovick, DS, et al. 14, October 4, 1984, New England Journal of Medicine, Vol. 311, pp. 874-877.

3. A Prospective Study of Sudden Cardiac Death among Children and Young Adults. Bagnall, RD, et al. 25, June 23, 2016, New England Journal of Medicine, Vol. 374, pp. 2441-2552.

4. Burden of sudden cardiac death in persons aged 1 to 49 years: nationwide study in Denmark. Risgaard, B, et al. 2, April 2014, Circulation, Arrhythmia and Electrophysiology, Vol. 7, pp. 205-211.

5. Lifetime Risk for Sudden Cardiac Death in the Community. Bogle, BM, et al. 7, July 2016, Journal of the American Heart Association, Vol. 5, pp. 1-10.

6. Suddent Unexpected Death in Persons Less Than 40 Years of Age. Drory, $\mathbf{Y}$ and Turetz, $\mathbf{Y}$ et al. 1991, American Journal of Cardiology, Vol. 68, p. 1388.

7. Causes of sudden cardiac death in young Australians. Doolan, A, Semsarian, $C$ and Langlois, N. 3, February 2, 2004, Medical Journal of Australia, Vol. 180, pp. 110-112.

8. Sudden death in the young: what do we know about it and how to prevent? van der Werf, C, van Langen, IM and Wilde, AA. 2010, Circ Arrhythm Electrophysiol, Vol. 3, pp. 96-104.

9. Death of the Teaching Autopsy. O'Grady, G. 2003, Britsh Medical Journal , Vol. 327, p. 802.

10. Exome analysis-based molecular autopsy in cases of sudden unexplained death in the young. Bagnall, RD, et al. 4, April 2014, Heart Rhythm, Vol. 11, pp. 655-662.

11. Sudden death in young adults: an autopsy-based series of a population undergoing active surveillance. Eckart, RE, et al. 12, 2011, Journal of the American College of Cardiology, Vol. 58, pp. 1254-1261.

12. Neonatal Autopsies: A 10-Year Experience. Kumar, P, et al. 1, January 2000, Archives of Pediatrics and Adolscent Medicine, Vol. 154, pp. 38-42.

13. Routine CT scan combined with preliminary examination as a new method in determining the need for autopsy. Bedford, PJ. 2012, Forensic Science, Medicine and Pathology, Vol. 8, pp. 390-394.

14. Post-mortem MRI versus conventional autopsy in fetuses and children: a prospective validation study. Thayyil, S, et al. 2013, The Lancet, Vol. 382, pp. 223-233.

15. Post-mortem computed tomography: Technical principles and recommended parameter settings for high-resolution imaging. Gascho, D, Thali, MJ and Niemann, T. 1, 2018, Medicine, Science and the Law, Vol. 58, pp. 70-82.

16. Modern post-mortem imaging: an update on recent developments. Grabherr, S, et al. 2, 2017, Forensic Sciences Research, Vol. 2, pp. 52-64.

17. Wood, R, Bassett, $\mathrm{K}$ and Foester, $\mathbf{V}$ et al. 1.5 Tes/a Magnetic Resonance Imaging Scanners Compared with 3.0 Tesla Magnetic Resonance Imaging Scanners: Systematic Review of Clinical Effectiveness: Pilot Project. Canadian Agency for Drugs and Technologies in Health. Ottawa : s.n., 2011.

18. Post-mortem Cardiovascular Magnetic Resonance Imaging in Fetuses and Children A Masked Comparison Study With Conventional Autopsy. Taylor, A, Sebire, N and Ashworth, M et al. 19, May 13, 2014, Circulation, Vol. 129, pp. 1937-1944.

19. Comparison of diagnostic performance for perinatal and paediatric post-mortem imaging: CT versus MRI. Arthurs, 0, et al. 2016, European Radiology, Vol. 26, pp. 23272336. 
20. Comparison of conventional autopsy and magnetic resonance imaging in determining the cause of sudden death in the young. Puranik, R, et al. 2014, Journal of Cardiovascular Magentic Resonance, Vol. 16, p. 44.

21. Diagnostic accuracy of post-mortem magnetic resonance imaging in fetuses, children and adults: a systematic review. Thayyil, S, et al. 1, July 2010, European Journal of Radiology, Vol. 75, pp. 142-148.

22. Post-mortem examinations using magnetic resonance imaging: four year review of a working service. Bisset, R, et al. 1998, British Medical Journal, Vol. 317, p. 1450.

23. Post-mortem Whole-Body Magnetic Resonance Imaging as an Adjunct to Autopsy: Preliminary Clinical Experience. Patriquin, L, et al. 2001, journal of Magnetic Resonance Imaging, Vol. 13, pp. 277-287.

24. Post-mortem imaging as an alternative to autopsy in the diagnosis of adult deaths: a validation study. Roberts, IS, et al. 9811, January 14, 2012, Lancet, Vol. 379, pp. 136-142. 25. New perspectives on the prevalence of hypertrophic cardiomyopathy. Semsarian, $\mathbf{C}$, et al. 12, March 31, 2015, Journal of the American College of Cardiology, Vol. 65, pp. 12491254.

26. Sudden death in young athletes. Maron, BJ. 2003, New Engladn Journal of Medicine, Vol. 349, pp. 1064-1075.

27. Classification and histological, immunohistochemical, and molecular diagnosis of inflammatory myocardial disease. Basso, C, et al. 6, November 2013, Heart Failure Reviews, Vol. 18, pp. 673-681.

28. Genetics of hypertrophic cardiomyopathy after 20 years: clinical perspectives. Maron, BJ, Maron, MS and Semsarian, C. 8, August 21, 2012, Journal of the American College of Cardiology, Vol. 60, pp. 705-715.

29. HRS/EHRA expert consensus state- ment on the state of genetic testing for the channelopathies and cardiomyo- pathies this document was developed as a partnership between the Heart Rhythm Society (HRS) and the European Heart Rhythm Association (EHRA). Ackerman, MJ, Priori, SG and Willems, S et al. 2011, Heart Rhythm, Vol. 8, pp. 1308-39.

30. Genetic testing for inherited cardiac disease. Wilde, AA and Behr, ER. 2013, Nature Reviews Cardiology, Vol. 10, pp. 571-583.

31. Prospective, population-based long QT molecular autopsy study of post-mortem negative sudden death in 1 to 40 year olds. . Skinner, JR, Crawford, J and Smith, W. 2011, Heart Rhythm , Vol. 8, pp. 412-419.

32. Cardiac channel molecular autopsy: insights from 173 consecutive cases of autopsynegative sudden unexplained death referred for post-mortem genetic testing. Tester, DJ, et al. 6, June 2012, Mayo Clinic Proceedings, Vol. 87, pp. 524-539.

33. Sudden cardiac death in the young: the molecular autopsy and a practical approach to surviving relatives. Semsarian, C, Ingles, J and Willde, AA. 21, June 1, 2015, European Heart Journal, Vol. 36, pp. 1290-1296.

34. Evaluation of post-mortem MDCT and MDCT-angiography for the investigation of sudden cardiac death related to atherosclerotic coronary artery disease. Michaud, $\mathrm{K}$, et al. 7, 2011, International Journal of Cardiovascular Imaging, Vol. 28, pp. 1807-1822.

35. Targeted post-mortem computed tomography cardiac angiography: proof of concept. Saunders, S, et al. 2011, International Journal of Legal Medicine, Vol. 125, pp. 609-616. 36. Post-mortem magnetic resonance imaging dealing with low temperature objects. Kobayashi, T, et al. 2010, Magnetic Resonance Medical Science, Vol. 9, pp. 101-108. 
37. Post mortem radiology: A new sub-speciality? O'Donnell, C and Woodford, N. 2008, Clinical Radiology, Vol. 63, pp. 1189-1194.

38. Post-mortem computedtomography: Technical priniciples and remmended parameter settings for high-resolution imaging. Gascho, D, Thali, MJ and Niemann, T. 1, 2018, Vol. 58, pp. 70-82.

39. Prevalence of arrhythmias during 24-hour electrocardiographic monitoring and exercise testing in patients with obstructive and nonobstructive hypertrophic cardiomyopathy.

Savage, DD, et al. 5, May 1979, Circulation, Vol. 59, pp. 866-875.

40. Syncope and risk of sudden death in hypertrophic cardiomyopathy. Spirito, P, et al. 13, April 7, 2009, Circulation, Vol. 119, pp. 1703-1710.

41. How should hypertrophic cardiomyopathy be classified? Elliott, P and Mckenna, WJ. 1, February 17, 2009, Controversies in Cardiovascular Genetics, Vol. 2, pp. 87-89.

42. Occurrence and frequency of arrhythmias in hypertrophic cardiomyopathy in relation to delayed enhancement on cardiovascular magnetic resonance. Adabag, AS, et al. 14, April 8, 2008, Journal of the American College of Cardiology, Vol. 51, pp. 1369-1374.

43. The molecular genetic basis for hypertrophic cardiomyopathy. Marian, AJ and Roberts, R. 2001, Journal of Molecular Cell Cardiology , Vol. 33, pp. 655-670.

44. Post-mortem CT and MRI: appropriate post-mortem imaging appearances and changes related to cardiopulmonary resuscitation. Offiah, CE and Dean, J. 1058, 2016, The British Journal of Radiology, Vol. 89.

45. Sudden cardiac death in the young: the molecular autopsy and a practical approach to surviving relatives. Semsarian, C, Ingles, J and Wilde, AA. 21, June 1, 2015, European Heart Journal, Vol. 36, pp. 1290-1296.

46. Update on the Diagnosis and Management of Hypertrophic Cardiomyopathy. Semsarian,

C. March 27, 2018, Heart, Lung and Circulation, Vol. 3, pp. 276-279.

47. Risk stratification for sudden cardiac death in hypertrophic cardiomyopathy: systematic review of clinical risk markers. I, Chritianns, et al. 3, March 1, 2010, EP Eurospace, Vol. 12, pp. 313-321. 
Figure 1: Post-mortem Magnetic Resonance Imaging with Correlating Histopathology Acute myocardial infarction; (A-B) Axial T2 STIR and short axis T2 STIR MR images demonstrating regional hyperintense signal of the distal lateral wall, consistent with acute myocardial infarction/oedema. (C) Movat pentachrome strain, 2x objective showing culprit left anterior descending coronary artery atherosclerosis with acute occlusive thrombus. Arrhythmogenic Right Ventricular Cardiomyopathy; (A - B) 4 chamber and short axis b-FFE MR image demonstrating severe RV dilation. (C) H\&E stain, 10x objective showing right ventricular outflow tract focal fibrosis, scanty lymphocytic infiltration and extensive fatty infiltration.

Aortic Dissection; (A-B) 3-d "whole-heart" MR imaging reconstructed in axial and saggital planes demonstrating severe ascending aortic dilatation and aortic dissection posteriorly with an associated large haemopericardium. (C) Movat pentachrome stain, 10x objective of aorta showing marked cystic medial degeneration with large pools of acid mucin. 
Figure 1

A

Acute Myocardial Infarction

Arrhythmogenic Right Ventricular Cardiomyopathy (ARVC)

Aortic Dissection
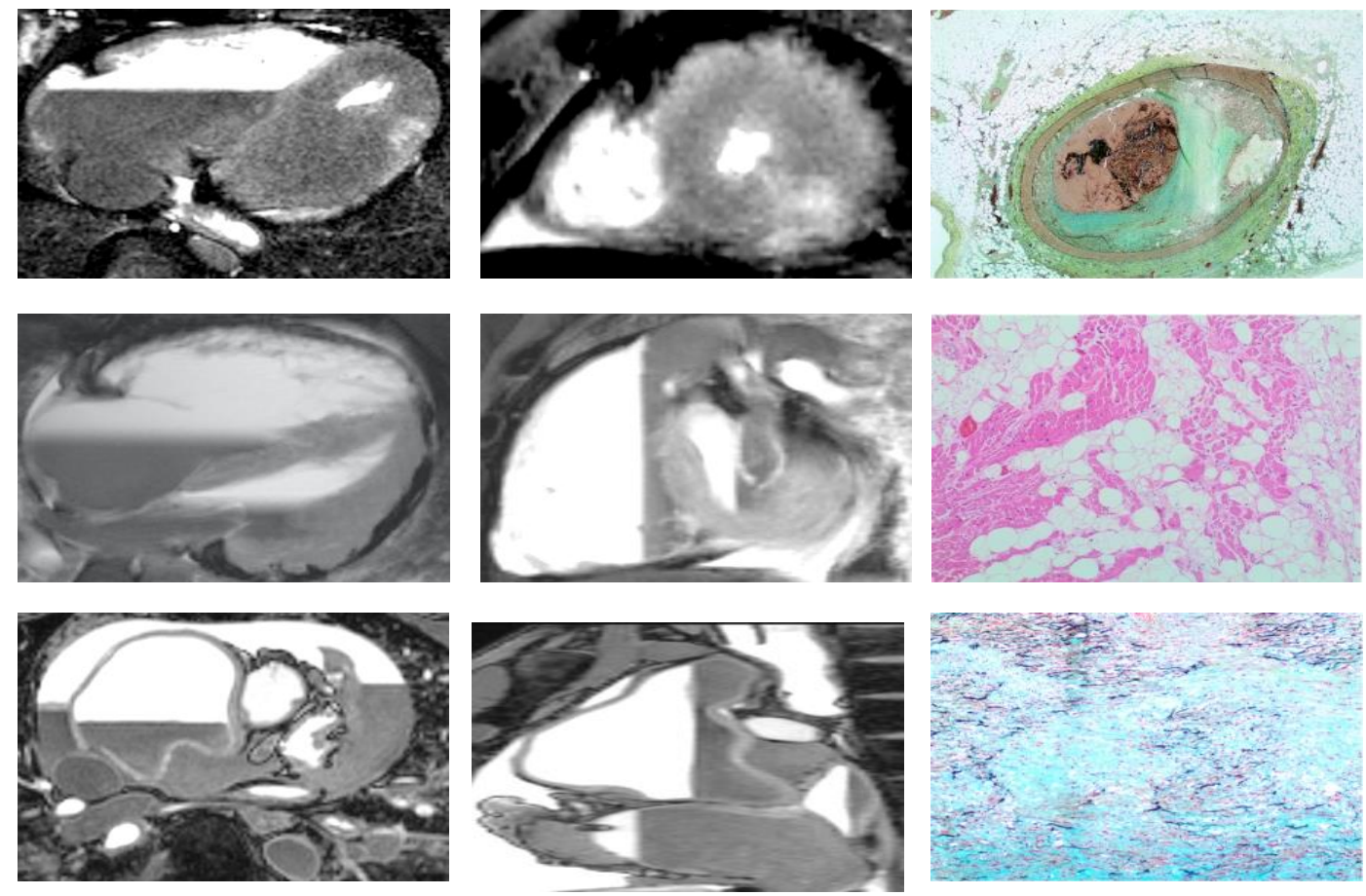
Table 1: Comparing Post-mortem Computed Tomography (pmCT) and Post-mortem Magnetic Resonance Imaging (pmMR)

\begin{tabular}{|l|c|c|}
\hline & pmCT & pmMR \\
\hline Availability & Good & Limited \\
\hline Maintenance Costs & High & Long \\
\hline Acquisition time (whole body) & Short & ro minutes \\
\hline Spatial Resolution & High minutes & High \\
\hline Indications & $\begin{array}{c}\text { Skeletal system } \\
\text { injuries/trauma, } \\
\text { detection of foreign } \\
\text { bodies, detection of } \\
\text { air/gas/fluid }\end{array}$ & $\begin{array}{c}\text { Natural deaths, excellent } \\
\text { soft tissue constrast for } \\
\text { solid organs }\end{array}$ \\
\hline
\end{tabular}

\begin{tabular}{|l|c|c|}
\hline Adult Cases & Preferred & Limited indications \\
\hline Coronary Artery Calcification & Yes & No \\
\hline Coronary stenosis/thrombosis & Possible & Potential role \\
\hline Acute myocardial infarction & No & \\
\hline
\end{tabular}

\begin{tabular}{|l|c|c|}
\hline Fetal and Paediatric cases & Limited indications & Preferred \\
\hline Anomalous Coronary Artery & Yes & No \\
\hline Hypertrophic cardiomyopathy & No & Yes \\
\hline Ventricular Cardiomyopathy & No & Yes \\
\hline Dilated Cardiomyopathy & Yes & Yes \\
\hline Marfan Syndrome & Yes & Use as a "rule out" test \\
for structural heart \\
Arrhythmogenic Conditions
\end{tabular}

